International Journal of Pure and Applied Mathematics

Volume 90 No. 2 2014, 153-164

ISSN: 1311-8080 (printed version); ISSN: 1314-3395 (on-line version)

url: http://www.ijpam.eu

doi: http://dx.doi.org/10.12732/ijpam.v90i2.5

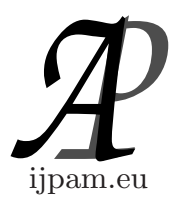

\title{
MATHEMATICAL MODEL OF MAGNETOMETRIC RESISTIVITY SOUNDING FOR A CONDUCTIVE \\ MEDIUM WITH A POSITIVELY SKEWED BULGE OVERBURDEN
}

\author{
Panyawat Haarsa \\ Department of Mathematics \\ Faculty of Science \\ Srinakharinwirot University \\ Bangkok, 10110, THAILAND
}

\begin{abstract}
The intention of this paper is consecrated to make known a mathematical model of magnetic response of a two layered earth model with a positively skewed bulge conductivity. The conductivity of ground is a function of depth. Integral expressions are applied, and the Taylor-series expansion and approximation are employed to discover the solution of the homogeneous differential equation. Numerical solutions are computed to present the conduct of the magnetic field graphically. The responses of magnetic field show some significance happened as a result of the depth and a positively skewed bulge conductivity ground profile.
\end{abstract}

AMS Subject Classification: 86A25

Key Words: magnetic field, positively skewed bulge, Chave's algorithm, Hankel transform, Taylor series

\section{Introduction}

In geophysical exploration, the magnetometric resistivity is opted to be one of the most utilized methods. The procedure is found upon the estimation of

Received: August 29, 2013

(c) 2014 Academic Publications, Ltd. url: www.acadpubl.eu 
a low level and low frequency magnetic fields connected with a non inductive current flow from the electrodes in the ground. Yooyuanyong and Sripanya [8] obtained and performed the solution of the steady state magnetic field caused a DC current source in three types of heterogeneous earth models. Chen and Oldenburg [3] acquired the magnetic field directly from solving a boundary value problems which was related to the approach used by Edward. Chaladgarn and Yooyuanyong [10] derived a mathematical model of magnetometric resistivity sounding for a conductive host with a bulge overburden.

In this paper, we acquaint a mathematical model and methods for studying the structure of the earth which has a two layered conductive model. We consider the ground having the conductivity which is presented by $\sigma_{o}(z)=$ $\left(\sigma_{0}+z\right) e^{-b z}, 0 \leq z \leq d$, where $\sigma_{0}$ is a positive constant, $b$ is constant, $d$ is the thickness of overburden. The conductivity of host medium is a constant denoted by $\sigma_{h}(z)=\sigma_{0}, z \geq d$, on the second layer. The conductivity profile in this research is unlike from the models used by Chen and Oldenburg [3] and Chaladgarn and Yooyuanyong [10].

\section{Derivation and Solution of Magnetic Field}

The general steady state Maxwell's equation in the frequency domain [3] can be used to define the magnetic field for this problem particularly

$$
\nabla \times \vec{E}=\overrightarrow{0}
$$

and

$$
\nabla \times \vec{H}=\sigma \vec{E}
$$

Where $\vec{E}$ is the vector electric field, $\vec{H}$ is the vector magnetic field, $\sigma$ is the conductivity of the medium in Siemens per meter $(S / m)$ which is supposed just to be a function of $z$, and $\nabla$ is the del operator. By getting rid of $\vec{E}$ from the above equations and leading to get

$$
\nabla \times \frac{1}{\sigma} \nabla \times \vec{H}=\overrightarrow{0} .
$$

It can be shown in a cylindrical coordinates $(r, \phi, z)$ as

$$
\frac{1}{r}\left[\frac{\partial}{\partial \phi} \frac{1}{r}\left(\frac{1}{\sigma} \frac{\partial r H_{\phi}}{\partial r}-\frac{1}{\sigma} \frac{\partial H_{r}}{\partial \phi}\right)-\frac{\partial}{\partial z}\left(\frac{1}{\sigma} \frac{\partial H_{r}}{\partial z}-\frac{1}{\sigma} \frac{\partial H_{z}}{\partial r}\right)\right] \hat{e}_{r}
$$




$$
\begin{aligned}
& +\left[\frac{\partial}{\partial z} \frac{1}{r}\left(\frac{1}{\sigma} \frac{\partial H_{z}}{\partial \phi}-\frac{1}{\sigma} \frac{\partial r H_{\phi}}{\partial z}\right)-\frac{\partial}{\partial r} \frac{1}{r}\left(\frac{1}{\sigma} \frac{\partial r H_{\phi}}{\partial r}-\frac{1}{\sigma} \frac{\partial H_{r}}{\partial \phi}\right)\right] \hat{e}_{\phi} \\
& +\frac{1}{r}\left[\frac{\partial}{\partial r}\left(\frac{1}{\sigma} \frac{\partial H_{r}}{\partial z}-\frac{1}{\sigma} \frac{\partial H_{z}}{\partial r}\right)-\frac{\partial}{\partial \phi} \frac{1}{r}\left(\frac{1}{\sigma} \frac{\partial H_{z}}{\partial \phi}-\frac{1}{\sigma} \frac{\partial r H_{\phi}}{\partial z}\right)\right] \hat{e}_{z}=\overrightarrow{0}
\end{aligned}
$$

Where $H_{r}, H_{\phi}, H_{z}$ are the components of $\vec{H}$ in $\hat{e}_{r}, \hat{e}_{\phi}, \hat{e}_{z}$ directions, respectively. Since the problem is axisymmetric, and $\vec{H}$ has only the azimuthal component in cylindrical coordinates. We use $H$ to symbolize the azimuthal component $H_{\phi}$ in the following derivations. By simplifying equation (4), this leads to

$$
\frac{\partial^{2} H}{\partial z^{2}}+\sigma \frac{\partial}{\partial z}\left(\frac{1}{\sigma}\right) \frac{\partial H}{\partial z}+\frac{\partial^{2} H}{\partial r^{2}}+\frac{1}{r} \frac{\partial H}{\partial r}-\frac{1}{r^{2}} H=0
$$

Where $\sigma$ is a function of the depth $z$ which is provided by $\sigma(z)$. The Hankel transform [4] is acquainted and defined by

$$
\tilde{H}(\lambda, z)=\int_{0}^{\infty} r H(r, z) J_{1}(\lambda r) d r .
$$

and

$$
H(r, z)=\int_{0}^{\infty} \lambda \tilde{H}(\lambda, z) J_{1}(\lambda r) d \lambda .
$$

Where $J_{1}$ is the Bessel function of the first kind of order one, and $\lambda$ is the Hankel variable. Applying the transformation to equation (5), we derive

$$
\frac{\partial^{2} \tilde{H}}{\partial z^{2}}+\sigma \frac{\partial}{\partial z}\left(\frac{1}{\sigma}\right) \frac{\partial \tilde{H}}{\partial z}-\lambda^{2} \tilde{H}=0
$$

Because the electrode is in the overburden which the end of the electrode is placed at $z=d$ so that a magnetic field will be isolated into two parts. The magnetic field is come from the ground layer that can be explained by the general solutions of equation (8) and the magnetic field arising from probe sources $H$, which is only one element. It can be interpreted by the Ampere's law $[8]$ as

$$
H(r, z)=\frac{I}{2 \pi r}
$$


Where $I$ is the current at the probe on the ground surface. Equation (6) yields

$$
\tilde{H}(\lambda, z)=\int_{0}^{\infty} r\left(\frac{I}{2 \pi r}\right) J_{1}(\lambda r) d r=\frac{I}{2 \pi \lambda} .
$$

By applying the inverse Hankel transform to the solution of equation (8), we have the magnetic fields in each layer which satisfies the following boundary conditions $[1,7]$.

1. The vertical component of the current density must be zero at the ground $\operatorname{surface}(z=0)$,

$$
\left.\sigma_{1}(z) E_{1}^{z}\right|_{z=0}=0 .
$$

where $E_{1}^{r}$ is the vertical component of the electric field in overburden.

2. The azimuthal component of the magnetic field have to be continuous on each of the boundary planes in the earth,

$$
\lim _{z \rightarrow d^{-}} \tilde{H}_{1}(\lambda, z)=\lim _{z \rightarrow d^{+}} \tilde{H}_{2}(\lambda, z) .
$$

Where $H_{1}$ and $H_{2}$ are magnetic field in the first and second layer, in the order.

3. The radial component of the electric field which is given by $E^{r}$ and have to be continuous on each of the boundary planes in the earth,

$$
\lim _{z \rightarrow d^{-}} \widetilde{E}_{1}^{r}(\lambda, z)=\lim _{z \rightarrow d^{+}} \widetilde{E}_{2}^{r}(\lambda, z) .
$$

Where $E_{1}^{r}$ is the radial component of the electric fields in the first layer, and $E_{2}^{r}$ is the radial component of the electric fields in the second layer. To find out the radial and vertical component of the electric field associated with the azimuthal of the magnetic field, we expand equation (2) and obtain [10]

$$
\begin{aligned}
E= & \left(\frac{1}{r \sigma} \frac{\partial H_{z}}{\partial \phi}-\frac{1}{\sigma} \frac{\partial H_{\phi}}{\partial z}\right) e_{r}+\left(\frac{1}{\sigma} \frac{\partial H_{r}}{\partial z}-\frac{1}{\sigma} \frac{\partial H_{z}}{\partial r}\right) e_{\phi} \\
& +\left(\frac{1}{r \sigma} \frac{\partial}{\partial r}\left(r H_{\phi}\right)-\frac{1}{r \sigma} \frac{\partial H_{r}}{\partial \phi}\right) e_{z}=0 .
\end{aligned}
$$




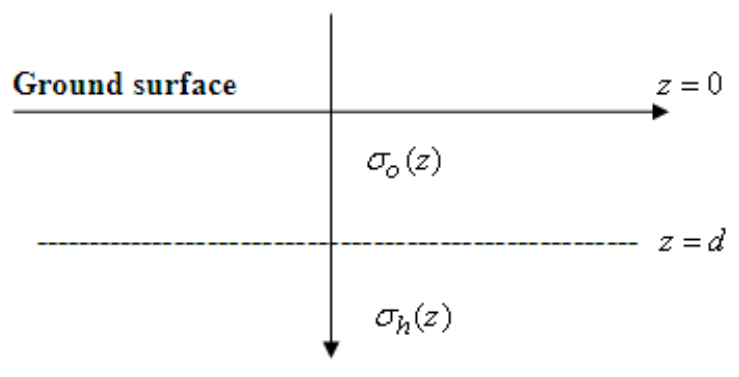

Figure 1: A geometric 2-layered conductive earth model.

Since the problem is axisymmetric and $\vec{H}$ has only the azimuthal component in cylindrical coordinates, we use $H$ to represent the azimuthal component $H_{\phi}$ in the above derivations and yields

$$
E^{r}=-\frac{1}{\sigma} \frac{\partial H}{\partial z}, \quad E^{z}=-\frac{1}{r \sigma} \frac{\partial}{\partial r}(r H)
$$

Equation (6) can be applied and directed to

$$
\widetilde{E}_{r}(\lambda, z)=\int_{0}^{\infty} r\left[-\frac{1}{\sigma(z)} \frac{\partial}{\partial z} H(r, z)\right] J_{1}(\lambda r) d r=-\frac{1}{\sigma(z)} \frac{\partial}{\partial z} \widetilde{H}(\lambda, z) .
$$

For this geometric model, a two-layered earth model is studied which the interface between the layers is a plane collimate to the ground surface. A point source of direct current $I$ is placed into the overburden for which the tip of the electrode is located at $z=d$. The first layer, the conductivity of overburden is presented by $\sigma_{o}(z)=\left(\sigma_{0}+z\right) e^{-b z}, 0 \leq z \leq d$, where $\sigma_{0}$ is a positive constant, $b$ is constant, $d$ is the thickness of overburden. The conductivity of host medium is a constant denoted by $\sigma_{h}(z)=\sigma_{0}, z \geq d$, on the second layer.

By substituting $\sigma_{o}(z)$ and $\sigma_{h}(z)$ into equation (8), we derive the magnetic fields in overburden and conductive host medium as

$$
\frac{\partial^{2} \tilde{H}_{1}}{\partial z^{2}}-\left(b-\frac{1}{x+\sigma_{0}}\right) \frac{\partial \tilde{H}_{1}}{\partial z}-\lambda^{2} \tilde{H}_{1}=0 .
$$

and

$$
\frac{\partial^{2} \tilde{H}_{2}}{\partial z^{2}}-\lambda^{2} \tilde{H}_{2}=0
$$




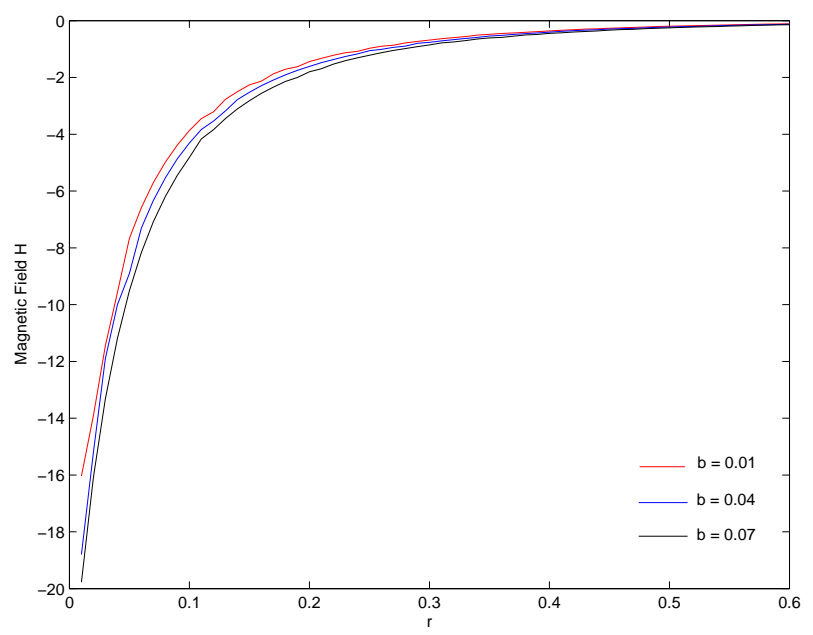

Figure 2: Curves of magnetic field $H$ versus $r$ for a two layered model with a positively skewed bulge conductivity ground profile where $z=$ $1 m, d=5 m, \sigma_{0}=1 S m^{-1}, b=0.01 m^{-1}, b=0.04 m^{-1}, b=0.07 m^{-1}$.

The solution of the homogenous differential equation (15) can be solved by using the Taylor-series method, and the auxiliary equation can be applied to find the solution of equation (16). Hence, we have

$$
\tilde{H}_{1}(\lambda, z)=A_{11}\left(1+\frac{\lambda^{2} z^{2}}{2}\right)+A_{12}\left(z+\frac{(b+1)}{2} z^{2}\right)+\frac{I}{2 \pi \lambda} .
$$

and

$$
\tilde{H}_{2}(\lambda, z)=B_{11} e^{-\lambda(z-d)}+B_{12} e^{\lambda(z-d)} .
$$

Where $A_{11}, A_{12}, B_{11}$ and $B_{12}$ are arbitrary constants which can be determined by using the boundary conditions. As $z \rightarrow \infty$, we require $B_{12}=0$ and equation (18) becomes

$$
\tilde{H}_{2}(\lambda, z)=B_{11} e^{-\lambda(z-d)}
$$

We make use of the first boundary condition into equation (9), we get

$$
\sigma_{1}(z)\left(\frac{1}{r \sigma_{1}(z)} \frac{\partial}{\partial r}\left(r H_{1}(r, z)\right)=0\right)
$$




\begin{tabular}{|c|c|c|c|}
\hline $\mathrm{r}$ & $\mathrm{z}=\mathbf{1}$ b=0.5 d=1 & $\mathrm{z}=\mathbf{1}$ b=0.5 d=1.5 & $\mathrm{z}=\mathbf{1}$ b=0.5 d=3 \\
\hline 0 & -4.012768337 & -3.373865898 & -4.973494928 \\
\hline 0.01 & -3.501088754 & -2.915343435 & -4.29945034 \\
\hline 0.02 & -3.075162969 & -2.56307044 & -3.741532055 \\
\hline 0.03 & -2.717128434 & -2.243723096 & -3.275153166 \\
\hline 0.04 & -2.413516356 & -1.993344014 & -2.881844257 \\
\hline 0.05 & -2.154019343 & -1.762931124 & -2.515259762 \\
\hline 0.06 & -1.930643627 & -1.572714287 & -2.261303062 \\
\hline 0.07 & -1.737114701 & -1.408202657 & -2.014672929 \\
\hline 0.08 & -1.568453449 & -1.269990134 & -1.800897854 \\
\hline 0.09 & -1.420669135 & -1.139908842 & -1.614598092 \\
\hline 0.1 & -1.290533882 & -1.029883404 & $-1.45 E+00$ \\
\hline 0.11 & -1.175414846 & $-9.33 E-01$ & $-1.29 E+00$ \\
\hline 0.12 & -1.073147848 & $-8.50 E-01$ & $-1.18 E+00$ \\
\hline 0.13 & $-9.82 E-01$ & $-7.70 E-01$ & $-1.06 E+00$ \\
\hline 0.14 & $-9.00 E-01$ & $-7.01 E-01$ & $-9.68 E-01$ \\
\hline 0.15 & $-8.27 E-01$ & $-6.40 E-01$ & $-8.69 E-01$ \\
\hline 0.16 & $-7.61 E-01$ & $-5.85 E-01$ & $-7.90 E-01$ \\
\hline 0.17 & $-7.01 E-01$ & $-5.35 E-01$ & $-7.27 E-01$ \\
\hline 0.18 & $-6.47 E-01$ & $-4.90 E-01$ & $-6.54 E-01$ \\
\hline 0.19 & $-5.98 E-01$ & $-4.49 E-01$ & $-6.03 E-01$ \\
\hline 0.2 & $-5.53 E-01$ & $-4.12 E-01$ & $-5.50 E-01$ \\
\hline & & & \\
\hline
\end{tabular}

Figure 3: Chave's algorithm [2] is used for numerical calculating the inverse Hankel transform by setting $\sigma_{0}=1 S \mathrm{~m}^{-1}, z=1 \mathrm{~m}, b=0.5 \mathrm{~m}^{-1}$, $d=1 m, d=1.5 m, d=3 m$.

For the reason that $\sigma_{1}(z)$ and $r$ are not zero, then

$$
\frac{\partial}{\partial r}\left(r H_{1}(r, z)\right)=0 \text {. }
$$

or

$$
r H_{1}(r, z)=\int_{0}^{\infty} \frac{\partial}{\partial r}\left(r H_{1}(r, z)\right) d r=\int_{0}^{\infty} 0 d r=0 .
$$

As $r>0$, then $H_{1}(r, z)=0$ and $\widetilde{H}_{1}(r, z)=\int_{0}^{\infty} r H_{1}(r, z) J_{1}(\lambda r) d r$.

Accordingly,

$$
\begin{aligned}
A_{11}\left(1+\frac{\lambda^{2} z^{2}}{2}\right) & +A_{12}\left(z+\frac{(b+1)}{2} z^{2}\right)+\frac{I}{2 \pi \lambda} \\
& =\int_{0}^{\infty} r(0) J_{1}(\lambda r) d r=0 .
\end{aligned}
$$


Since no electric current across at the air-earth interface, that is $I=0$, hence

$$
A_{11}\left(1+\frac{\lambda^{2} z^{2}}{2}\right)+A_{12}\left(z+\frac{(b+1)}{2} z^{2}\right)=0 .
$$

At $z=0$, we gain $A_{11}=0$. By using the second boundary condition, we attain

$$
\lim _{x \rightarrow d^{-}}\left[A_{12}\left[z+\frac{(b+1)}{2} z^{2}\right]+\frac{I}{2 \pi \lambda}\right]=\lim _{x \rightarrow d^{+}} B_{11} e^{-\lambda(z-d)} .
$$

This follows,

$$
B_{11}=A_{12}\left[d+\frac{(b+1)}{2} d^{2}\right]+\frac{I}{2 \pi \lambda} .
$$

By making use of the third second boundary condition, we obtain

$$
\lim _{z \rightarrow d^{-}} \frac{1}{\left(\sigma_{0}+z\right) e^{-b z}} \frac{\partial}{\partial z}\left[A_{12}(1+(b+1) z)\right]=\lim _{z \rightarrow d^{+}} \frac{\lambda}{\sigma_{0}} \frac{\partial}{\partial z}\left[B_{11} e^{-\lambda(z-d)}\right] .
$$

Therefore,

$$
A_{12}=\frac{I}{\pi}\left[\frac{\left(\sigma_{0}+d\right)}{2 \sigma_{0}[1+(b+1) d] e^{\lambda(z-d)+b d}-\lambda\left(\sigma_{0}+d\right)\left[2 d+(b+1) d^{2}\right]}\right] .
$$

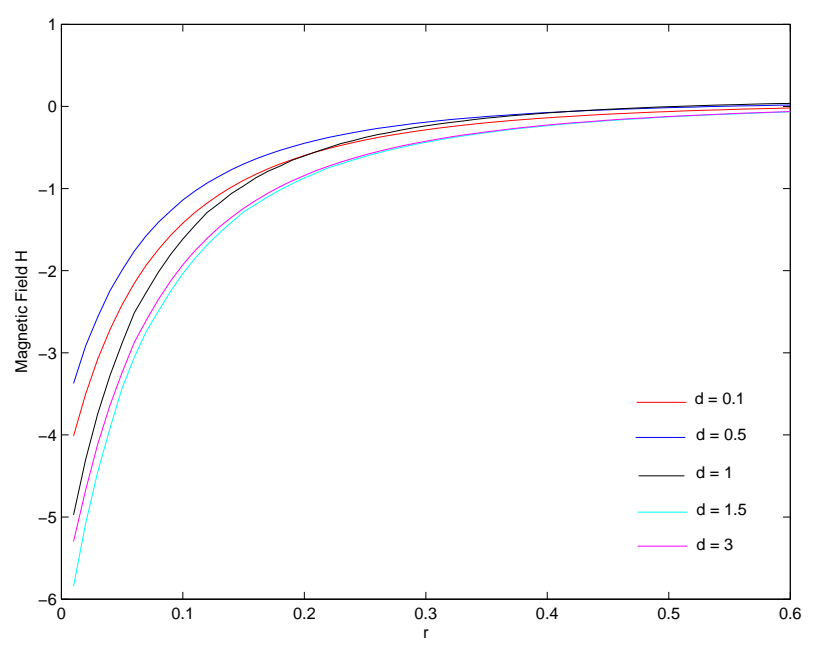

Figure 4: Curves of magnetic field $H$ versus $r$ for a two layered model with a positively skewed bulge conductivity ground profile where $z=$ $1 \mathrm{~m}, b=0.5 \mathrm{~m}^{-1}, \sigma_{0}=1 \mathrm{Sm}^{-1}, d=0.1 \mathrm{~m}, d=0.5 \mathrm{~m}, d=1 \mathrm{~m}$, $d=1.5 m, d=3 m$ 


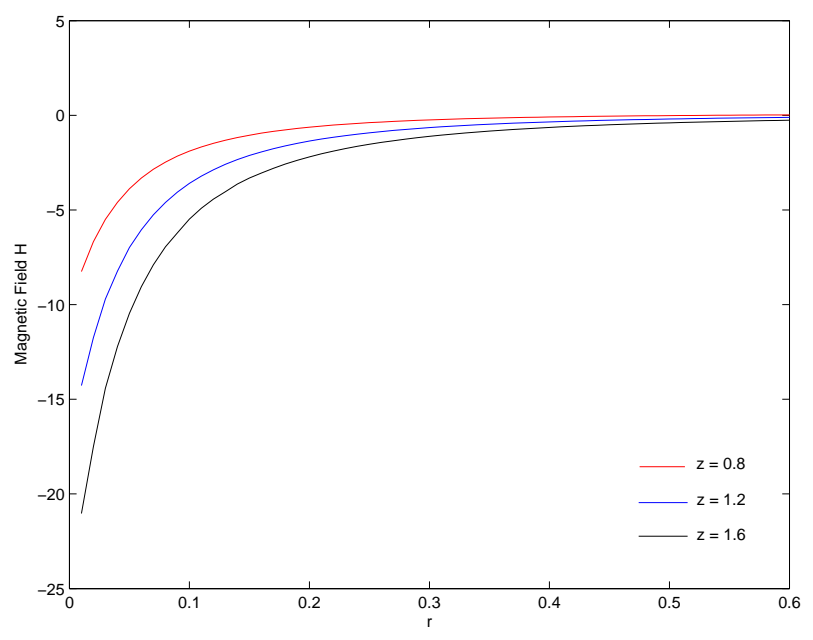

Figure 5: Curves of magnetic field $H$ versus $r$ for a two layered model with a positively skewed bulge conductivity ground profile where $d=$ $1 \mathrm{~m}, \sigma_{0}=1 S \mathrm{~m}^{-1}, b=0.5 \mathrm{~m}^{-1}, z=0.8 \mathrm{~m}, z=1.2 \mathrm{~m}, z=1.6 \mathrm{~m}$.

and

$$
\begin{aligned}
B_{11}= & \frac{\sigma_{0} e^{\lambda(z-d)+b d} I}{\pi \lambda} \\
& \times\left[\frac{1+(b+1) d}{2 \sigma_{0}[1+(b+1) d] e^{\lambda(z-d)+b d}-\lambda\left(\sigma_{0}+d\right)\left[2 d+(b+1) d^{2}\right]}\right] .
\end{aligned}
$$

Consequently, by using the inverse Hankel transforms, the magnetic fields in overburden and conductive host medium can be described, in the order, as

$$
H_{1}(r, z)=\int_{0}^{\infty}\left[\frac{I \kappa_{22}+2 \lambda I\left(\sigma_{0}+d\right)}{2 \pi \kappa_{22}}\right] \times\left[z+\frac{(b+1) z^{2}}{2}\right] J_{1}(\lambda r) d \lambda .
$$

and

$$
H_{2}(r, z)=\frac{\sigma_{0}}{\pi} \int_{0}^{\infty} \kappa_{11}[1+(b+1) d] e^{-\lambda(z-d)} J_{1}(\lambda r) d \lambda .
$$

where $\kappa_{11}=\frac{e^{\lambda(z-d)+b d} I}{\kappa_{22}}$ and $\kappa_{22}=2 \sigma_{0}[1+(b+1) d] e^{\lambda(z-d)+b d}-\lambda\left(\sigma_{0}+d\right)[2 d+$ $\left.(b+1) d^{2}\right]$. 


\begin{tabular}{|c|c|c|c|}
\hline$r$ & $d=2 \quad b=1 \quad z=2$ & $d=2 \quad b=1 \quad z=2.5$ & $d=2 \quad b=1 \quad z=3$ \\
\hline 0 & -28.96040292 & -41.0690462 & -55.05992366 \\
\hline 0.01 & -23.16369804 & -32.93950161 & -44.2394668 \\
\hline 0.02 & -18.9025021 & -27.16943172 & -36.70819667 \\
\hline 0.03 & -15.68252111 & -22.42112016 & -30.21485399 \\
\hline 0.04 & -13.19291553 & -19.04989666 & -25.80795181 \\
\hline 0.05 & $-1.12 E+01$ & -16.14154977 & -21.82389704 \\
\hline 0.06 & $-9.66 E+00$ & $-1.39 E+01$ & -18.84815064 \\
\hline 0.07 & $-8.38 E+00$ & $-1.21 E+01$ & $-1.66 E+01$ \\
\hline 0.08 & $-7.33 E+00$ & $-1.07 E+01$ & $-1.44 E+01$ \\
\hline 0.09 & $-6.45 E+00$ & $-9.37 E+00$ & $-1.29 E+01$ \\
\hline 0.1 & $-5.71 E+00$ & $-8.37 E+00$ & $-1.13 E+01$ \\
\hline 0.11 & $-5.09 E+00$ & $-7.48 E+00$ & $-1.02 E+01$ \\
\hline 0.12 & $-4.55 E+00$ & $-6.71 E+00$ & $-9.20 E+00$ \\
\hline 0.13 & $-4.09 E+00$ & $-6.05 E+00$ & $-8.31 E+00$ \\
\hline 0.14 & $-3.69 E+00$ & $-5.48 E+00$ & $-7.53 E+00$ \\
\hline 0.15 & $-3.35 E+00$ & $-4.95 E+00$ & $-6.86 E+00$ \\
\hline 0.16 & $-3.04 E+00$ & $-4.54 E+00$ & $-6.26 E+00$ \\
\hline 0.17 & $-2.77 E+00$ & $-4.15 E+00$ & $-5.74 E+00$ \\
\hline 0.18 & $-2.54 E+00$ & $-3.81 E+00$ & $-5.27 E+00$ \\
\hline 0.19 & $-2.33 E+00$ & $-3.48 E+00$ & $-4.83 E+00$ \\
\hline 0.2 & $-2.14 E+00$ & $-3.23 E+00$ & $-4.49 E+00$ \\
\hline & & & \\
\hline
\end{tabular}

Figure 6: Chave's algorithm [2] is used for numerical calculating the inverse Hankel transform by setting $d=2 \mathrm{~m}, \sigma_{0}=1 S \mathrm{~m}^{-1}, b=1 \mathrm{~m}^{-1}$, $z=2 m, z=2.5 m, z=3 m$.

\section{Summing-up and Approaching Work}

In this work, we give the method to study the structure of the earth by constructing a mathematical model. We consider a two layered earth model having a positively skewed bulge conductivity profile on the first layer which is given by $\sigma_{o}(z)=\left(\sigma_{0}+z\right) e^{-b z}, 0 \leq z \leq d$, where $\sigma_{0}$ is a positive constant, $b$ is constant, $d$ is the thickness of overburden. The conductivity of host medium, $z \geq d$, is a constant denoted by $\sigma_{h}(z)=\sigma_{0}$ on the second layer. We derive the magnetic fields by formulating the problem. The Taylor-series expansion and approximation are used to find the solution of the homogeneous differential equation. Chave's algorithm is operated for numerical calculating the inverse transform of the magnetic field reply. In the experiments, we impose the value of $\sigma_{0}=1 S \mathrm{~m}^{-1}$ and the current $I=1$ ampere throughout the trial while the values of $b, z$, and $d$ are varied. The magnetic fields were expressed in term of mathematical expressions and plotted as shown in figures. The curves are shown the conduct of the magnetic field against source-receiver spacing $r$. As the overburden thickness increase, the magnetic fields enlarge instantaneously 


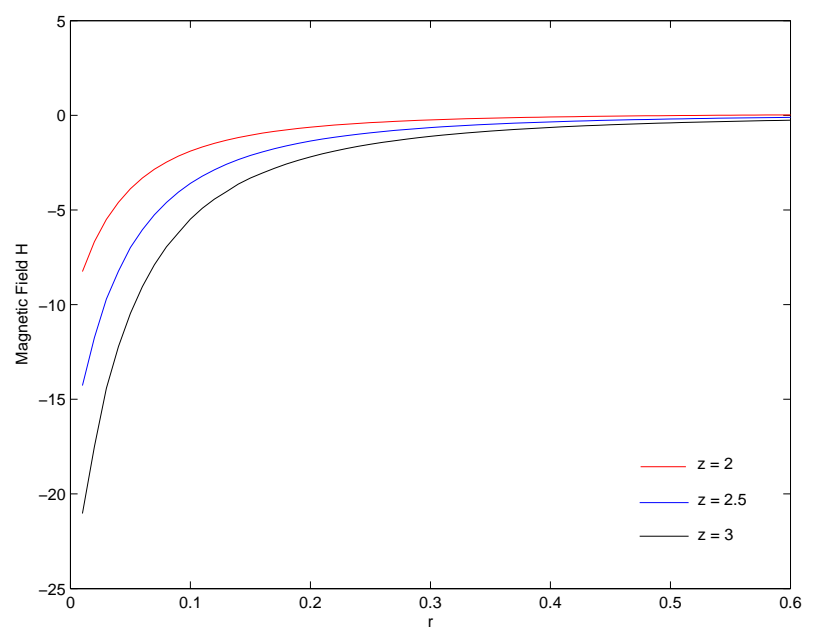

Figure 7: Curves of magnetic field $H$ versus $r$ for a two layered model with a positively skewed bulge conductivity ground profile where $d=$ $2 m, \sigma_{0}=1 S m^{-1}, b=1 m^{-1}, z=2 m, z=2.5 m, z=3 m$.

and conduce to zero as we anticipated. It is resulted not only by the depth $z$ but also by a positively skewed bulge conductivity. We noticed that the differences of the curves shown in figures are relied on the variation of conductivity. In order to find the proper values for all the parameters in this model, the inverse problem is required for a further study, and the optimization will be our alternative.

\section{References}

[1] B. Banerjee, B.J. Sengupta, B.P. Pal, Apparent resistivity of a multilayered earth with a layer having exponentiality varying conductivity, Geophys. Prospecting, 28 (1980), 435-452.

[2] A.D. Chave, Numerical integration of related Hankel transforms by quadrature and continued fraction expansion, Geophysics, 48 (1983), 1671-1686.

[3] J.Chen, D.W. Oldenburg, Magnetic and electrical fields of direct currents in a layered earth, Expl. Geophys, 77 (2004), 157-163.

[4] I. Ali, S. Kalla, A generalized Hankel transform and its use for solving cer- 
tain partial differential equations, J. Austral Math. Soc. Ser B, 41 (1999), 105-117.

[5] W. Sripanya, Magnetic Field of Direct Current in Heterogeneous Media, M.Sc. Thesis, Silpakorn University, Thailand (2005).

[6] R.N. Edwords, Downhole magnetometric resistivity technique for electrical sounding beneath a conductive surface layer, Geophysics, 53 (1988), 528536.

[7] C.H. Stoyer, J.R. Wait, Resistivity probing of an "exponential" earth with a homogeneous overburden, Geoexploration, 15 (1977), 11-18.

[8] S. Yooyuanyong, W. Sripanya, Magnetic field of direct current in heterogeneous ground, Songklanakarin J. Sci. Technol, 29 (2007), 565-573.

[9] S. Yooyuanyong, W. Sripanya, Mathematical modeling of magnetometric resistivity sounding earth structures, Thai J. Maths., 3, No. 2 (2005), 249258.

[10] T. Chaladgarn, S. Yooyuanyong, Mathematical model of magnetometric resistivity sounding for a conductive host with a bulge overburden, Applied Math. Sci., 7, No-s: 5-8 (2013), 335-348.

[11] P. Haarsa, Electromagnetic response on a half-space earth with a positively skewed bulge conductivity, Int. J. Applied Math. Stat., 42, No. 12 (2013), 324-329.

[12] P. Haarsa, S. Pothat, Mathematical model of electromagnetic response from a positively kewed bulge ground structure, Applied Math. Sci., 7, No. 101 (2013), 5013-5023. 